\title{
HEAT TRANSFER IN THIN METAL FILM MODELED BY BOLTZMANN TRANSPORT EQUATION AND A TWO-TEMPERATURE MODEL
}

\author{
Korczak A.*, Dziatkiewicz J..*, Piasecka-Belkhayat A..**, Majchrzak E..***
}

\begin{abstract}
In the paper a description of heat transfer in a one-dimensional metal films is considered. The Boltzmann transport equation and a two-temperature model supplemented by appropriate boundary and initial conditions are applied to analyze the thermal process in a heated thin metal film. The problem considered is solved by the lattice Boltzmann method and the finite difference method respectively. The internal heat source is given in two different ways. In the first way, the internal heat source is considered a constant value, while in the second way an exponential function which simulates irradiation using a laser pulse is used. In the final part of the paper numerical examples of comparison of two methods and conclusions are presented.
\end{abstract}

\section{Keywords: Boltzmann transport equation, Two-temperature model, Heat transfer, Lattice Boltzmann} method, Finite difference method.

\section{Introduction}

In metals heat transport is generally realized by two kinds of heat carriers: electrons and quanta of lattice vibrations called phonons. It is important to take into account these both energy carriers when creating mathematical model of this phenomena in the nanoscale. The use of the system of coupled Boltzmann transport equations and the two-temperature model, here proposed, allow to determine the heat exchange in the electron gas and the metal lattice, which is not the case when using the popular Fourier equation.

\section{Methods}

In the paper the Boltzmann transport equation and two-temperature model are considered. A thin metal film is irradiated by laser. The internal heat source is given in two ways. In the first one, a constant heat source $Q(t)=$ const is considered. The second way takes into account the temporal variation of the laser pulse approximated by a form of exponential function (Lee, 2011).

$$
Q(t)=I_{0} e^{-\beta t}
$$

where $I_{0}$ is the peak power intensity of the laser pulse, $\beta$ is the laser pulse parameter that determines the shape of the pulse.

\subsection{The lattice Boltzmann method and the Boltzmann transport equation}

The unsteady BTEs transformed into equivalent energy density equations for the 1D coupled model with two kinds of carriers (e-electrons and l-lattice vibrations called phonons) can be written using the formulas (Escobar, 2006 and Hopkins, 2009):

\footnotetext{
* Asst. Prof. Anna Korczak, PhD.: Department of Computational Mechanics and Engineering, Silesian University of Technology; Akademicka 2A; 44-100, Gliwice; PL, anna.korczak@polsl.pl

** Asst. Prof. Jolanta Dziatkiewicz, PhD.: Department of Computational Mechanics and Engineering, Silesian University of Technology; Akademicka 2A; 44-100, Gliwice; PL, jolanta.dziatkiewicz@polsl.pl

*** Assoc. Prof. Alicja Piasecka-Belkhayat, PhD.: Department of Computational Mechanics and Engineering, Silesian University of Technology; Akademicka 2A; 44-100, Gliwice; PL, alicja.piasecka-belkhayat@polsl.pl

${ }^{* * * *}$ Prof. Ewa Majchrzak, PhD.: Department of Computational Mechanics and Engineering, Silesian University of Technology; Akademicka 2A; 44-100, Gliwice; PL, ewa.majchrzak@polsl.pl
} 


$$
\begin{aligned}
& \frac{\partial e_{e}(x, t)}{\partial t}+\mathbf{v}_{e} \cdot \nabla e_{e}(x, t)=-\frac{e_{e}(x, t)-e_{e}^{0}(x, t)}{\tau_{e}}+Q_{e}(x, t) \\
& \frac{\partial e_{l}(x, t)}{\partial t}+\mathbf{v}_{l} \cdot \nabla e_{l}(x, t)=-\frac{e_{l}(x, t)-e_{l}^{0}(x, t)}{\tau_{l}}+Q_{l}(x, t)
\end{aligned}
$$

where $e_{e}, e_{l}$ are the energy densities, $e_{e}^{0}, e_{l}^{0}$ are the equilibrium energies densities, $\mathbf{v}_{e}, \mathbf{v}_{l}$ are the frequency-dependent propagation speed, $\tau_{e}, \tau_{l}$ are the relaxation times, $t$ denotes the time and $Q_{e}, Q_{l}$ are the energies source related to an unit of volume for electrons and lattice respectively.

The electron and phonon energy densities at their equivalent nonequilibrium temperatures are given by the formulas (Escobar, 2006)

$$
\begin{gathered}
e_{e}\left(T_{e}\right)=\left(n_{e} \frac{\pi^{2}}{2} \frac{k_{b}^{2}}{\varepsilon_{F}}\right) T_{e}^{2} \\
e_{l}\left(T_{l}\right)=\left(\frac{9 \eta_{l} k_{b}}{\Theta_{D}^{3}} \int_{0}^{\Theta_{D} / T_{l}} \frac{z^{3}}{\exp (z)-1} \mathrm{~d} z\right) T_{l}^{4}
\end{gathered}
$$

where $\Theta_{D}$ is the Debye temperature of the solid, $k_{b}$ is the Boltzmann constant, $T_{e}, T_{l}$ are the temperature for electrons and phonons respectively, while $n_{e}$ is the electron density and $\eta_{p h}$ is the phonon density. The electron and phonon energy sources are calculated using the following expressions

$$
Q_{e}(x, t)=Q(t)-G\left(T_{e}(x, t)-T_{p h}(x, t)\right), Q_{p h}(x, t)=G\left(T_{e}(x, t)-T_{p h}(x, t)\right)
$$

where $G$ is the electron-phonon coupling factor which characterizes the energy exchange between electrons and phonons. Eqs. (2) and (3) should be supplemented by the initial and boundary conditions.

\subsection{The finite difference method and a two-temperature model}

Two-temperature model describing the temporal and spatial evolution of the lattice and electrons temperatures $\left(T_{l}\right.$ and $\left.T_{e}\right)$ in the irradiated metal can be written (Chen, 2001) (1D problem)

$$
\begin{gathered}
C_{e}\left(T_{e}\right) \frac{\partial T_{e}(x, t)}{\partial t}=-\frac{\partial q_{e}(x, t)}{\partial t}-G\left[T_{e}(x, t)-T_{l}(x, t)\right]+Q(t) \\
C_{l} \frac{\partial T_{l}(x, t)}{\partial t}=-\frac{\partial q_{l}(x, t)}{\partial t}+G\left[T_{e}(x, t)-T_{l}(x, t)\right]
\end{gathered}
$$

where $T_{e}(x, t), T_{l}(x, t)$ are the temperatures of electrons and lattice, respectively, $C_{e}\left(T_{e}\right), C_{l}$ are the volumetric specific heats, $G$ is the electron-phonon coupling factor which characterizes the energy exchange between electrons and phonons, $Q(t)$ is the source function associated with the irradiation.

Instead of the classical Fourier law the following formulas are introduced

$$
\begin{gathered}
q_{e}(x, t)+\tau_{e} \frac{\partial q_{e}(x, t)}{\partial t}=-\lambda_{e}\left(T_{e}, T_{l}\right) \frac{\partial T_{e}(x, t)}{\partial x} \\
q_{l}(x, t)+\tau_{l} \frac{\partial q_{l}(x, t)}{\partial t}=-\lambda_{l} \frac{\partial T_{l}(x, t)}{\partial x}
\end{gathered}
$$

where $\lambda_{e}\left(T_{e}, T_{l}\right), \lambda_{l}$ are the thermal conductivities of electrons and lattice, respectively, $\tau_{e}$ is the relaxation time of free electrons in metals, $\tau_{l}$ is the relaxation time in phonon collisions.

For low laser intensity the following relationships describing the electrons thermal capacity and volumetric specific heat are widely used (Chen, 2001 and Lin, 2008)

$$
\lambda_{e}\left(T_{e}, T_{l}\right)=\lambda_{0} \frac{T_{e}}{T_{l}}, C_{e}\left(T_{e}\right)=A T_{e}
$$


where $\lambda_{0}$ and $A$ are the material constants (Majchrzak, 2012). For low laser intensity $\lambda_{l}, C_{l}$, and $G$ are also constant values depended on material.

To solve the problem formulated the algorithm basing on the finite difference method is used (Majchrzak, 2012). A staggered grid is introduced in which the temperature nodes $i=0,2,4, \ldots, N$ and the heat fluxes nodes $j=1,3, \ldots ., N-1$ are introduced. It means that $T_{i}^{f}=T(i h, f \Delta t)$ and $q_{j}{ }^{f}=q(j h, f \Delta t)$, where $h$ is the mesh size, $\Delta t$ is the time step $f=0,1,2, \ldots, F$.

\section{Results of computations}

As a numerical example the heat transport in a gold thin film of the thickness $L=100 \mathrm{~nm}$ has been analysed. The following input data have been introduced: the relaxation times $\tau_{e}=0.04 \mathrm{ps}, \tau_{p h}=0.8 \mathrm{ps}$, the Debye temperature $\Theta_{D}=170 \mathrm{~K}$, the peak power intensity of the laser pulse $I_{0}=2 \cdot 10^{13} \mathrm{~W} / \mathrm{m}^{2}$, the coupling factor $\mathrm{G}=2.3 \cdot 10^{16} \mathrm{~W} / \mathrm{m}^{3} \mathrm{~K}$, the boundary conditions of the $2^{\text {nd }}$ type on the both edges $q_{b 1}^{k}(0, t)=q_{b 2}^{k}(L, t)=0$, the initial temperature $T_{0}=300 \mathrm{~K}$. The lattice step $\Delta x=1 \mathrm{~nm}$ and the time step $\Delta t=0.001 \mathrm{ps}$ have been assumed. Three values of the laser pulse parameter $\beta$ was taken into account: 1) $\left.0.5 .10^{13} \mathrm{~s}^{-1} ; 2\right) 10^{13} \mathrm{~s}^{-1}$; 3) $1.5 .10^{13} \mathrm{~s}^{-1}$ (see Fig. 1b).
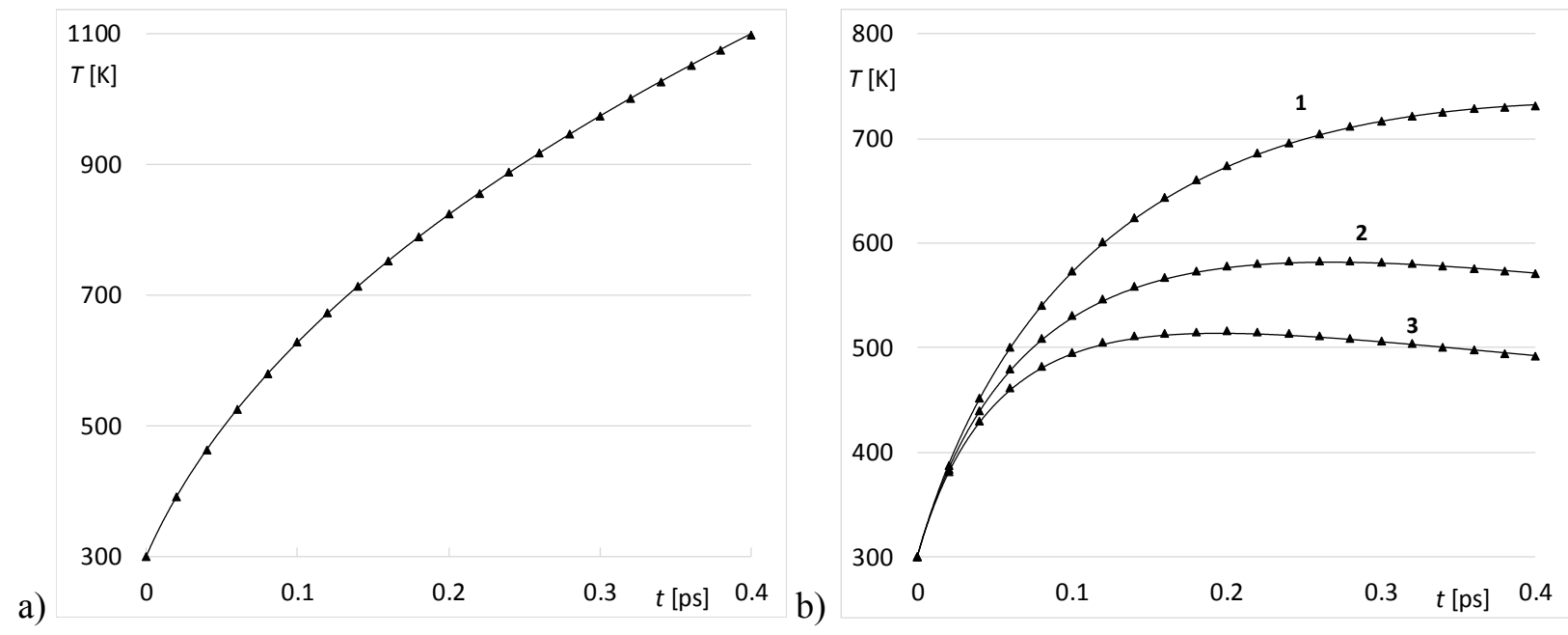

Fig. 1: Heating curves for $x=0$ : a) $Q(t)=$ const; b) $Q(t)=I_{0} e^{-\beta t}$.

Tab. 1: Comparison of temperatures in node $x=0, \beta=10^{13} \mathrm{~s}^{-1}$.

\begin{tabular}{|c|c|c|c|c|c|c|c|}
\hline time & FDM & LBM & $\delta[\%]$ & time & FDM & LBM & $\delta[\%]$ \\
\hline 0.04 & 440.0907 & 438.3789 & 0.38897 & 0.24 & 582.5611 & 580.8897 & 0.28691 \\
\hline 0.08 & 508.7353 & 506.5825 & 0.42317 & 0.28 & 582.7515 & 581.5039 & 0.21409 \\
\hline 0.12 & 546.5965 & 544.3312 & 0.41444 & 0.32 & 580.2610 & 579.5346 & 0.12519 \\
\hline 0.16 & 567.5201 & 565.3244 & 0.38689 & 0.36 & 576.0237 & 575.9115 & 0.01948 \\
\hline 0.2 & 578.2466 & 576.2557 & 0.3443 & 0.4 & 570.6550 & 571.2458 & 0.1035 \\
\hline
\end{tabular}

In Figs. 1a and $1 \mathrm{~b}$ heating curves obtained using Boltzmann transport equation (continuous line) and two temperature model (triangles) is shown. The consistency of the results is very high. Tab. 1 presents estimated error between these two methods. In Fig. 2 the distributions of heat source function for three different values of $\beta:$ 1) $0.5 .10^{13} \mathrm{~s}^{-1}$; 2) $10^{13} \mathrm{~s}^{-1}$; 3) $1.5 .10^{13} \mathrm{~s}^{-1}$ are presented.

To compare the results of the two presented approaches the relative error $\delta$ is defined (see Tab. 1)

$$
\delta=\left|\frac{\mathbf{F D M}-\mathbf{L B M}}{\mathbf{F D M}}\right| \cdot 100 \%
$$




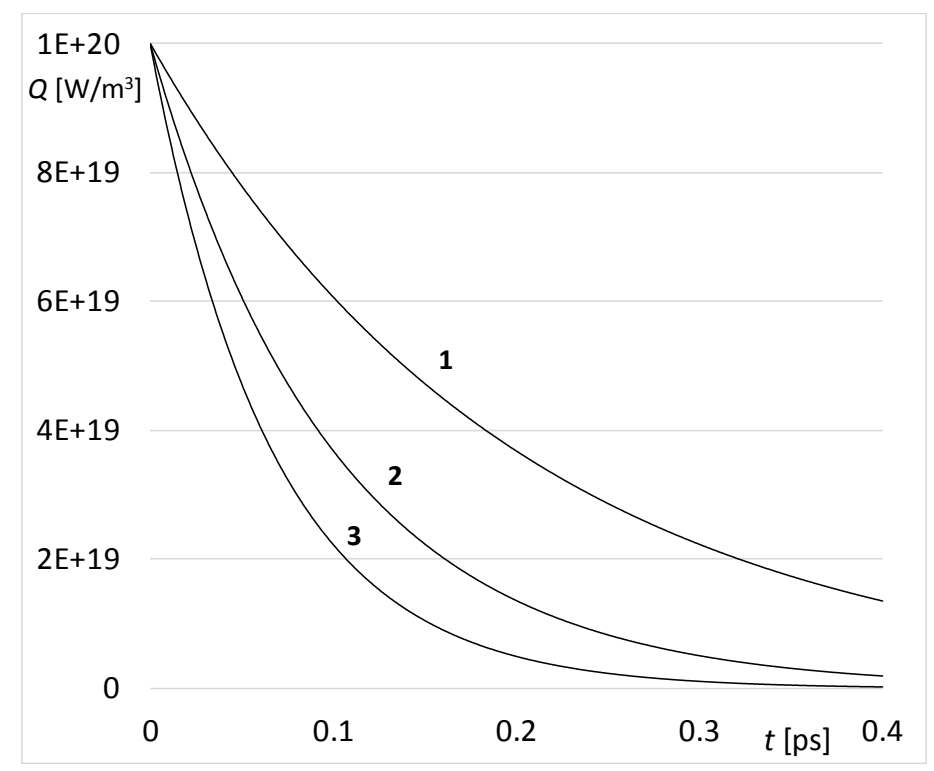

Fig. 2: Heat source function.

\section{Conclusions}

In the paper the temperatures obtained using two different mathematical models: the Boltzmann transport equation and the two-temperature model were compared. Numerical methods: the lattice Boltzmann method and the finite difference method respectively were used to solve them. The results obtained when applying the two types of heat sources are comparable.

\section{Acknowledgement}

The research is funded from the projects Silesian University of Technology, Faculty of Mechanical Engineering.

\section{References}

Chen, J.K., Beraun, J.E. (2001) Numerical study of ultrashort laser pulse interactions with metal films, Numerical Heat Transfer, Part A, 40, pp. 1-20.

Escobar, R.A., Ghai, S.S., Jhon, M.S. and Amon, C.H. (2006) Multi-length and time scale thermal transport using the lattice Boltzmann method with application to electronics cooling, Journal of Heat and Mass Transfer, 49, pp. 97-107.

Hopkins, P.E. and Norris, P.M. (2009) Contribution of Ballistic Electron Transport to Energy Transfer During Electron-Phonon Nonequilibrium in Thin Metal Films, Journal Heat Transfer, 131, 4, 043208 pp. 1-8.

Lee, J. B., Kang, K. and Lee, S. H. (2011) Comparison of Theoretical Models of Electron-Phonon Coupling in Thin Gold Films Irradiated by Femtosecond Pulse Lasers, Materials Transactions, 52, 3, pp. 547-553.

Lin, Z., Zhigilei, L.V. (2008) Electron-phonon coupling and electron heat capacity of metals under conditions of strong electron-phonon nonequilibrium, Physical Review, B 77, pp. 075133-1-075133-17.

Majchrzak, E., Dziatkiewicz, J. (2012) Identification of electron-phonon coupling factor in a thin metal film subjected to an ultrashort laser pulse, Computer Assisted Methods in Engineering and Science, 19, pp. 383-392. 